\title{
Intoxicação por Sorhgum halepense (Poaceae) em bovinos no semi-árido ${ }^{1}$
}

\author{
J. Escarião da Nóbrega $\mathrm{Jr}^{2}$, Franklin Riet-Correa ${ }^{3}$, Rosane M.T. Medeiros ${ }^{3}$ \\ e Antônio F.M. Dantas ${ }^{3}$
}

\begin{abstract}
Nóbrega Jr J.E., Riet-Correa F., Medeiros R.M.T. \& Dantas A.F.M. 2006. [Poisoning by Sorghum halepense (Poaceae) in cattle in the Brazilian semiarid.] Intoxicação por Sorghum halepense (Poaceae) em bovinos no semi-árido. Pesquisa Veterinária Brasileira 26(4):201-204. Hospital Veterinário, CSTR, Universidade Federal de Campina Grande, Campus de Patos, 58700-000 Patos, PB, Brazil. E-mail: franklin.riet@pesquisador .com.br

An outbreak of poisoning by Sorghum halepense (L.) Pers. in cattle in the Brazilian semiarid is reported. Nine cattle were introduced 15 days after the first rains into a paddock where the plant was sprouting and had $25-30 \mathrm{~cm}$ high. Clinical signs of dyspnea, anxiety, muscular tremors and incoordination appeared 15 minutes after the animals began to graze. Two of them died within about 3 hours. The others recovered. Cyanotic mucosa, dark muscles, lung edema and hemorrhages, and leaves of the plant in the rumen were observed at necropsy. The plant was positive for the picrosodic paper test. S. halepense from another farm of occurrence of the poisoning was transplanted into two pots. The picrosodic paper test was performed fortnightly in the plants growing in one of them. The plant was positive for cyanide in all growth stages; during the first 45 days the test was positive within 1 minute, but after day 60 within 3 minutes. Thirty days after the plant had been transplanted into one of the pots, it was administered to a goat at a dose of $11.8 \mathrm{~g} /$ $\mathrm{kg}$, causing clinical signs of $\mathrm{HCN}$ poisoning. The animal recovered after the intravenous administration of $5 \mathrm{~mL} / 10 \mathrm{~kg}$ body weight of a $20 \%$ sodium thiosulfate solution. In the Brazilian semiarid S. halepense is frequently found invading the border of ponds and irrigated crops, and is used as forage during the dry season.
\end{abstract}

INDEX TERMS: Poisonous plants, Sorghum halepense, cyanogenic glycosides, plant poisoning, cattle.

RESUMO.- Descreve-se um surto de intoxicação por Sorghum halepense (L.) Pers. no município de Santa Luzia, semi-árido da Paraíba. Nove bovinos foram colocados numa pastagem com $S$. halepense em fase de rebrota, com 25-30cm de altura, 15 dias após as primeiras chuvas. Sinais clínicos de dispnéia, ansiedade, tremores musculares e incoordenação apareceram 10-15 minutos após a ingestão. Dois animais morreram em um período aproximado de 3 horas. Na necropsia observaram-se congestão e cianose das mucosas, musculatura escura, pulmão com pontos hemorrágicos e edema, além de folhas da planta no rúmen. A planta foi positiva no teste do papel picrosódico. Mudas de S. halepense coletadas no

\footnotetext{
${ }^{1}$ Recebido em 24 de março de 2006.

Aceito para publicação em 17 de abril de 2006.

2 Médico Veterinário, Programa de Pós-Graduação em Medicina Veterinária de Pequenos Ruminantes Centro de Saúde e Tecnologia Rural, UFCG, Campus de Patos, 58700-000 Patos, PB.

${ }^{3}$ Centro de Saúde e Tecnologia Rural, Universidade Federal de Campina Grande (UFCG), Campus de Patos, 58700-000 Patos, Paraíba, Brasil. *Autor para correspondência: franklin.riet@pesquisador.cnpq.br
}

Município de Currais Novos, Rio Grande do Norte, onde havia históricos de uma intoxicação semelhante, foram plantadas em vasos e realizado o teste de papel picrosódico a cada 15 dias. A planta foi positiva para $\mathrm{HCN}$ durante todos os estágios, sendo que nos primeiros 45 dias a reação ocorreu dentro de 1 minuto e, posteriormente, em até 3 minutos. Trinta dias após ter sido plantada, a planta de um dos vasos foi administrada a um caprino causando sinais característicos de intoxicação cianídrica na dose de $11,8 \mathrm{~g} / \mathrm{kg}$ de peso do animal. $\mathrm{O}$ caprino se recuperou após tratamento com uma solução de tiossulfato de sódio a $20 \%$ na dose de $5 \mathrm{~mL} / 10 \mathrm{~kg}$. No semi-árido, S. halepense é freqüentemente encontrado como invasora em beiras de açudes e culturas irrigadas, sendo utilizado como forrageira, na época seca, em áreas de vazante.

TERMOS DE INDEXAÇÃO: Plantas tóxicas, Sorghum halepense, ácido cianídrico, intoxicação por planta, glicosídeos cianogênicos, bovinos.

\section{INTRODUÇÃO}

As plantas cianogênicas contêm como princípio ativo o ácido cianídrico (HCN), um líquido incolor, muito volátil, considerado como 
uma das substâncias mais tóxicas que se conhecem. Nos vegetais se encontra ligado a glicosídeos denominados cianogênicos e é liberado após a hidrólise dos mesmos. São registradas no mundo mais de 120 plantas consideradas cianogênicas (Radostits et al. 2000).

As plantas cianogênicas descritas no Brasil são Manihot esculenta (Euphorbiaceae) e várias espécies silvestres de Manihot, conhecidas como maniçobas (Amorim et al. 2005), Anadenanthera (Piptadenia) macrocarpa (angico) e Piptadenia viridiflora (espinheiro ou surucucu) da família Leguminosae Mimosoideae (Tokarnia et al. 1994a,b, 1999), Prunus sellowii da família Rosaceae (Saad \& Camargo 1967, Gava et al. 1992) e Cynodon dactylon (capim-Tifton) (Gava et al. 1998). Holocalyx glaziovii, também contém glicosideos cianogênicos e é tóxica experimentalmente, mas não há relatos de intoxicação espontânea (Armien et al. 1995).

No Brasil, o gênero Sorghum, que contém o glicosídeo cianogênico durrina (Knight \& Walter 2002), tem sido mencionado como tóxico (Méndez 1993, Tokarnia et al. 2000), mas não há publicações de surtos de intoxicação por este gênero. Sorghum halepense (sorgo-de-alepo, capim-massambará, capim-argentino, johnsongrass), conhecido em algumas áreas do semi-árido como capim de boi, é uma das plantas cianogênicas mais conhecidas no mundo, sendo considerada como planta invasora que causa prejuízos importantes para a pecuária. É uma planta nativa do sul da Ásia, Oriente Médio e áreas banhadas pelo mar Mediterrâneo. Foi introduzida no Brasil, no Estado do Rio Grande do Sul com sementes de linho, girassol, alfafa e sorgo provenientes da Argentina. Sua área de abrangência vem aumentando nas margens das rodovias e nas lavouras dos estados do Rio Grande do Sul, São Paulo e Paraná (Kissmann 1997). O nome sorgo de Alepo refere-se ao local de origem da planta na cidade de Aleppo na Síria, e o nome de capim Maçambará refere-se ao local onde foi introduzido aparentemente pela primeira vez no Brasil, numa estação ferroviária chamada Maçambará, no município de Itaquí, Rio Grande do Sul (Kissmann 1997). S. halepense é uma planta de cultivo perene, fortemente rizomatosa, cespitosa, de colmo ceroso, com pilosidade nos nós e um porte médio entre 1-2m de altura. As piores invasões ocorrem em terrenos periodicamente inundados, onde a dispersão das sementes se da primordialmente pela água. Um extenso sistema rizomatoso garante o repovoamento da área após períodos de condições adversas e a planta produz sustâncias alelopáticas que inibem o desenvolvimento de outras plantas (Kissmann 1997). No Nordeste, $S$. halepense é encontrado nas margens de açudes e culturas irrigadas, sendo utilizada como forrageira na época da seca.

Históricos de intoxicações por S. halepense (capim-de-boi) (Fig.1 e 2) são relatados por numerosos produtores da região do Seridó, no Rio Grande do Norte, e na região do Sabugi, no sertão Paraibano. Este trabalho tem como objetivo relatar um surto de intoxicação espontânea em bovinos ocorrida no semi-árido da Paraíba, assim como determinar a toxicidade de capim-de-boi coletado no Município de Currais Novos, Rio Grande do Norte.

\section{MATERIAL E MÉTODOS}

Um surto de intoxicação por Sorghum halepense foi diagnosticado no município de Santa Luzia no semi-árido da Paraíba. Os dados epidemiológicos e os sinais clínicos observados foram relatados pelo produtor. Uma necropsia foi realizada por um dos autores (J.E.N.Jr),

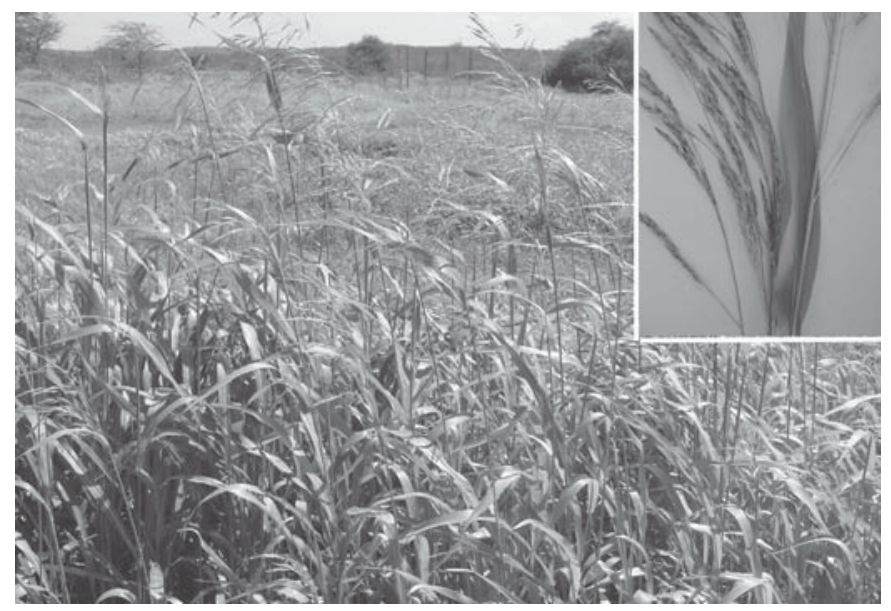

Fig.1. Sorghum halepense (capim-de-boi, sorgo-de-Alepo) na beira de um açude. Município de Currais Novos, Rio Grande do Norte. Inserido panícula com sementes.

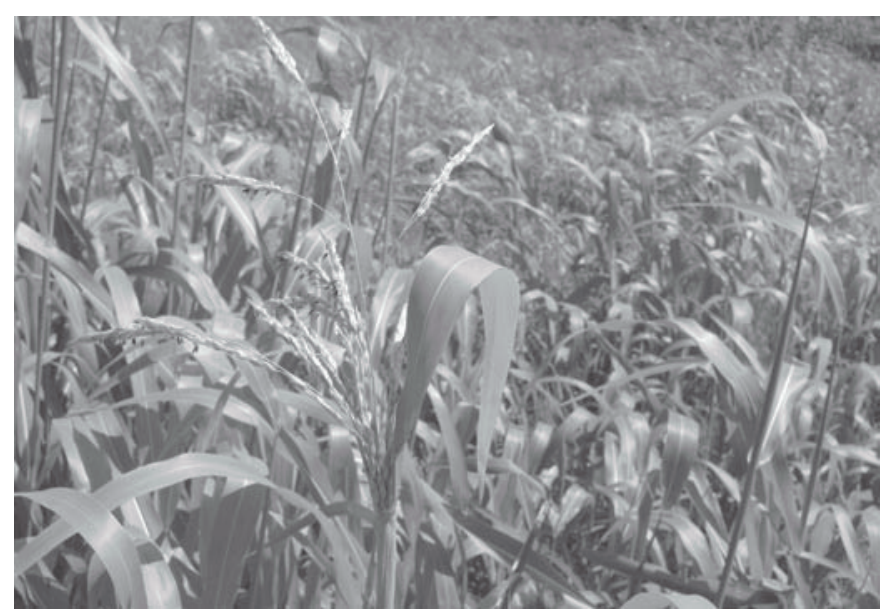

Fig.2. Sorghum halepense (capim-de-boi, sorgo-de-Alepo). Município de Currais Novos, Rio Grande do Norte.

aproximadamente 10 horas após a morte do animal. O teste de papel picrosódico para detectar presença de HCN (Tokarnia et al. 2000) foi realizado com a planta do local onde ocorreu o surto.

Mudas de $S$. halepense foram coletadas no município de Currais Novos, Rio Grande do Norte, e plantadas em dois vasos com $0,5 \mathrm{~m}$ de diâmetro por $0,5 \mathrm{~m}$ de profundidade, sendo regadas semanalmente. A cada 15 dias, desde a brotação até a frutificação, amostras das plantas de um dos vasos foram coletadas para realização do teste de papel picrosódico. Trinta dias após a plantação, a planta coletada de outro vaso foi administrada, imediatamente após a coleta, a um caprino macho da raça Moxotó, com 4 meses de idade, colocando pequenas quantidades na boca, até que apresentasse sinais clínicos característicos da intoxicação por HCN.

Exemplares da planta foram enviados para o Dr. Odaci F. de Oliveira, da Escola Superior de Agricultura de Mossoró para identificação botânica.

\section{RESULTADOS}

O surto ocorreu ema 18 de dezembro de 2005 em um rebanho de 20 bovinos de exploração leiteira, após chuvas ocorridas nos 
dias 4 e 6 de dezembro após um logo período seco, que nessa região estende-se de abril/maio a dezembro/janeiro. Nove bovinos, de diversas idades, provenientes de um pasto com muito pouca disponibilidade de forragem, foram soltos em uma área, ao redor de um açude, onde havia Sorghum halepense em estágio de rebrota, com aproximadamente $25-30 \mathrm{~cm}$ de altura.

Em um período de aproximadamente 15 minutos após o início da ingestão da planta, os animais apresentaram sinais de agitação, cambaleio e queda, timpanismo, depressão, desconforto abdominal, salivação espumosa, dispnéia com ofegância e taquipnéia, tremores musculares e incoordenação com andar cambaleante. Dois animais apresentaram sinais mais graves; deitavam-se e levantavam-se constantemente evoluindo para uma marcada depressão, decúbito e morte em um período de aproximadamente 3 horas após a ingestão. Os demais bovinos se recuperaram.

A planta coletada no Rio Grande do Norte e plantada em vasos resultou positiva para o teste do papel picrosódico durante todo o seu período vegetativo, sendo que nos primeiros 45 dias a reação ocorreu rapidamente, antes de 1 minuto, e posteriormente, em até 3 minutos. O último teste foi feito 150 dias após a plantação, quando a planta estava sementando.

O caprino que recebeu a planta com 30 dias de crescimento apresentou dispnéia severa e urinação freqüiente após a ingestão de dose de $11,8 \mathrm{~g} / \mathrm{kg}$ de peso do animal. Foi tratado com uma solução de tiossulfato de sódio a $20 \%$, intravenoso, na dose de $5 \mathrm{ml}$ por cada $10 \mathrm{~kg}$ e recuperou-se em alguns minutos.

A planta foi identificada como Sorghum halepense (L.) Pers. Segundo o Dr Odaci F. de Oliveira, os exemplares coletados, por terem espiguetas sem aristas correspondem a Sorghum halepense var. muticum (Hack. ex D.C.) Parodi, porém, ao longo do tempo, tem havido confusão na sistemática desta espécie, sendo possível que a característica "sem arista", isto é, mútica, tenha surgido devido à seleção em cultivos. Pela opinião do mencionado Botânico poderíamos utilizar a identificação em nível de variedade, ou adotar a mais geral, isto é, em nível de espécie. Considerando que não há nenhuma referência em relação à toxicidade das diferentes variedades optamos por utilizar a classificação de Sorghum halepense (L.) Pers.

\section{DISCUSSÃO}

O rápido aparecimento da intoxicação após o início da ingestão, os sinais clínicos característicos, o teste positivo para a prova do papel picrosódico e a reprodução experimental da intoxicação em um caprino confirmam o diagnóstico de intoxicação por ácido cianídrico. Além disso, a identificação da planta como Sorghum halepense, uma das plantas cianogênicas mais conhecidas na literatura (Dowling \& McKenzie 1993, Radostits et al. 2000, Knight \& Walter 2002), não deixa dúvidas em relação à causa da doença. Além da toxicidade da planta um fator muito importante para a ocorrência da intoxicação é o fato da mesma ser ingerida rapidamente, em um curto espaço de tempo, como ocorreu no surto descrito neste trabalho. A velocidade de ingestão das plantas cianogênicas é um dos fatores mais importantes para a ocorrência das intoxicações (Dowling \& McKenzie 1993). Outro fator importante para a ocorrência da intoxicação neste surto foi o fato de que quando os animais foram introduzidos, a planta tinha crescido rapidamente após as primeiras chuvas que determinaram o fim da estação seca. Períodos de rápido crescimento após períodos sem crescer é um dos fatores que aumenta o conteúdo de glicosídeos cianogênicos nas plantas (Radostits et al. 2000).

No semi-árido da Paraíba e Rio Grande do Norte Sorghum halepense é freqüentemente encontrado como invasora de culturas irrigadas e em culturas de vazante de forrageiras em beiras de açudes. Provoca intoxicação em bovinos quando ingerido na fase de rebrota, mas é utilizado como forragem após essa fase. Mediante o teste do papel picrosódico foi observado que a planta contêm maior quantidade de HCN até os 45-60 dias. Posteriormente continuou a ser positiva, mas num período maior de tempo, o que concorda com a informação dos produtores, de que nesta fase a planta não causa intoxicação.

Diversos produtores do Seridó Oriental e Seridó Ocidental no Rio Grande do Norte (Morais 2006) e da Paraíba conhecem a toxicidade do capim-de-boi e evitam sua utilização durante a fase de crescimento, sendo considerado como boa pastagem para a época da seca. Além de não utilizar $S$. halepense como forragem na época de crescimento, para evitar a intoxicação, é importante que os animais introduzidos na pastagem não estejam com fome, o que os leva a ingerir a planta rapidamente.

Agradecimentos.- Ao Dr Odaci O. de Oliveira Professor aposentado da Escola Superior de Agricultura de Mossoró pela identificação botânica da planta. Este projeto foi financiado pela Fundação de Amparo à Pesquisa da Paraíba/ Ministério de Ciência e Tecnologia, Edital 004/03 FAPESQ-MST, e pelo programa Institutos do Milênio Processo 420012/2005-2.

\section{REFERÊNCIAS}

Amorim S.L., Medeiros R.M.T. \& Riet-Correa F. 2005. Intoxicação experimental por Manihot glaziovii (Euphorbiaceae) em caprinos. Pesq. Vet. Bras. 25(3):179-187.

Armién A.G., Peixoto P.V., Döbereiner J. \& Tokarnia C. 1995. Intoxicação experimental por Holocalyx glaziovii (Leg.Mimosoideae) em bovinos. Pesq. Vet. Bras. 15:89-92.

Dowling R.M. \& McKenzie R.A. 1993. Poisonous Plants. A field guide. Department of Primary Industries, Queensland, Australia.164p.

Gava A., Stolf L., Neves D.S., Stolf O., Varaschim M.S. \& Ferreira F.M.M. 1992. Intoxicação experimental por Prunus sellowii (Rosaceae) em bovinos. Pesq. Vet. Bras. 12(1/2):1-4.

Gava A., Pilati C., Cristian J., Simões J. \& Simões L. 1998. Intoxicação cianogênica em bovinos alimentados com Tifton (Cynodon sp.). VIII CAMEV, Lages, Santa Catarina. 5p.

Kissmann K.G. 1997. Plantas Infestantes e Nocivas. $2^{\underline{a}}$ ed., BASF, São Paulo. p.751-760.

Knight A.P. \& Walter R.G. 2002. A Guide to Plant Poisoning of Animals in North America. Teton New Media, Jackson, Wyoming. 367p.

Mendez M.C. 1993. Intoxicação por plantas cianogênicas, p.279-284. In: Riet-Correa F., Méndez M.D.C \& Schild A.L. (ed.) Intoxicação por Plantas e Micotoxicoses em Animais Domésticos. Editorial Agropecuária Hemisfério Sur, Montevideo.

Morais D. 2006. Plantas tóxicas para ruminantes no Seridó Oriental e Occidental do Rio Grande do Norte. Tese de Mestrado, Curso de Medicina Veterinária de Pequenos Ruminantes, CSTR, UFCG, Patos, PB. (Em preparação) 
Radostits O.M., Gay C.C., Blood D.C. \& Hinchcliff K.W. 2000. Clínica Veterinária: um tratado de doenças de bovinos, ovinos, caprinos, suínos e equíídeos. 9 ed. $^{\mathrm{a}}$ ed Saunders, London, p.1631-1636.

Saad A.D. \& Camargo W.V.A. 1967. Intoxicação cianídrica em animais domésticos. Biológico, São Paulo, 33(10):211-220.

Tokarnia C.H., Döbereiner J. \& Peixoto P.V. 1994a. Aspectos clínico patológicos complementares da intoxicação por algumas plantas tóxicas brasileiras. Pes. Vet. Bras.14(4):111-122.
Tokarnia C.H., Peixoto P.V. \& Döbereiner J. 1994b. Intoxicação experimental por Piptadenia macrocarpa (Leg.Mimosideae) em bovinos. Pesq. Vet. Bras.12(2/3):57-63.

Tokarnia C.H., Peixoto P.V., Brito M.F., Duarte M.D. \& Brust L.A.C. 1999. Estudos experimentais com plantas cianogênicas em bovinos. Pesq. Vet. Bras. 19(2):84-90.

Tokarnia C.H., Döbereiner J. \& Peixoto P.V. 2000. Plantas Tóxicas do Brasil. Editora Helianthus, Rio de Janeiro, p.215-221. 